\title{
Performed Administrative Activity
}

National Cancer Institute

\section{Source}

National Cancer Institute. Performed Administrative Activity. NCI Thesaurus. Code C93355.

The completed activity within the context of a given study that is not directly related to the study's overarching hypothesis evaluation or testing, but is nonetheless essential to the efficient and/or effective coordination and execution of the study. 